\title{
Die Koalition der Unwilligen - Grundrisse für die Gesundheits- und Pflegepolitik der Großen Koalition
}

\section{FRANZ KNIEPS}

Franz Knieps ist Vorstandsvorsitzender des BKK Dachverbandes e.V. in Berlin. Er war von 2002 bis 2009 Abteilungsleiter „Krankenversicherung“ im Bundesministerium für Gesundheit und ist Herausgeber der Zeitschrift „Gesundheits- und Sozialpolitik“. Der Beitrag gibt die persönliche Auffassung des Verfassers wieder

Lange haben sie verhandelt, die Unterhändler von CDU, CSU und SPD über die Bildung einer von keiner Partei erwünschten Großen Koalition. Dabei ist der Koalitionsvertrag nicht gerade das, was man einen großen Wurf nennen würde. Doch viele Details, wie der Mindestlohn, die Erschwerung prekärer Beschäftigung, die doppelte Staatsbürgerschaft und manches mehr können sich sehen lassen. Der folgende Beitrag gibt einen Überblick darüber, was zu Gesundheit und Pflege vereinbart wurde und was aus Sicht eines kritischen Beobachters fehlt. Dabei ist zu berücksichtigen, dass ein Koalitionsvertrag die politischen Gemeinsamkeiten zu Beginn einer Legislaturperiode umreißt. Neue Herausforderungen suchen sich neue Lösungen. Bilanz ist immer am Ende einer gemeinsamen Wegstrecke zu ziehen.

\section{Akzente in der gesundheitlichen Versorgung - Neue Ansätze zu einer stärkeren Berücksichtigung von Qualität und Bedarfsgerechtigkeit}

„Im Zentrum unserer Gesundheitspolitik stehen Patientinnen und Patienten und die Qualität ihrer medizinischen Versorgung.“ Dieser Auftakt des Kapitels Gesundheit und Pflege zum Koalitionsvertrag für die 18. Legislaturperiode klingt banal, ist es aber nicht. Allzu oft spielen sich die Akteure des Gesundheitswesens mit ihren institutionellen und ökonomischen Interessen in den Vordergrund und drängen Bedarfe und Bedürfnisse der Bürgerinnen und Bürger in den Hintergrund. Es wäre bereits viel gewonnen, wenn die Vorrangstellung der Patientenorientierung bei allen Reformvorhaben der nächsten Jahre in der Praxis durchgesetzt würde. Dabei muss sich auch zeigen, ob die Fokussierung der Gesundheitspolitik auf die medizinische Versorgung nur ein sprachlicher Lapsus ist oder ob Gesundheitspolitik über das Medizinsystem hinausgreifen wird. Ideologisch überfrachtet ist das daran anknüpfende Bekenntnis zur Freiberuflichkeit. Es ist zu eng, wenn die Freiberuflichkeit von Ärzten und Therapeuten auf Niedergelassene beschränkt wäre, und zu weit gegriffen, wenn daraus die freie Arztwahl der Versicherten abzuleiten wäre. Der Kern einer wohl verstandenen Freiberuflichkeit ist vielmehr in der ärztlichen Ethik zu verorten, die im Zweifelsfall auch gegen die ökonomischen Interessen durchgesetzt werden muss, ohne der Illusion von der Medizin als ökonomiefreiem Raum zu erliegen.

Der Koalitionsvertrag geht davon aus, dass in der Realität der ambulanten 
ärztlichen und psychotherapeutischen Versorgung auch zehn Jahre nach der grundlegenden Analyse des Sachverständigenrats für die Entwicklung im Gesundheitswesen noch immer ein weitgehend ungeordnetes Nebeneinander von Über- und Unterversorgung besteht. Folglich enthält die Übereinkunft zahlreiche detaillierte Festlegungen, wie der flächendeckende Zugang der Bevölkerung zu differenzierten gesundheitlichen Angeboten unter schwierigeren Rahmenbedingungen gesichert werden soll. Der Instrumentenkasten ist erstaunlich vielfältig und wirft ideologische Beschränkungen aus der Vergangenheit über Bord. So sollen bürokratische Hindernisse bei der Zulassung abgebaut, Anreize für die Niederlassung in unterversorgten Gebieten verbessert, die Krankenhäuser weiter für die ambulante Behandlung geöffnet, Praxisnetze und Medizinische Versorgungszentren gefördert und die

\section{Eine zentrale Terminstelle bei der KV schafft noch keine zusätzlichen Behandlungskapazitäten.}

Bedingungen für die hausärztliche Versorgung verbessert werden. Der Überversorgung soll insbesondere durch die Verpflichtung (,soll“) zum Aufkauf nicht bedarfsnotwendiger Arztsitze durch die Kassenärztlichen Vereinigungen (KV) begegnet werden. Es verwundert wenig, wenn die Vertragsärzte in der Vertreterversammlung der Kassenärztlichen Bundesvereinigung (KBV) viele dieser Punkte prompt zurückweisen.

Besonderes Augenmerk widmen die Koalitionäre dem Problem (je nach Versicherungssystem - GKV oder PKV - unterschiedlich) langer Wartezeiten von Patientinnen und Patienten bei Vertragsärzten und Psychotherapeuten. $\mathrm{Ob}$ allerdings die Einrichtung einer zentralen Terminstelle bei der KV, die innerhalb einer Woche einen Facharzttermin vermitteln soll, und die Möglichkeit, nach vier Wochen vergeblichen Wartens ein Krankenhaus zur ambulanten Behandlung aufzusuchen, mehr als ein politisches Placebo darstellen, wird die künftige Realität erweisen müssen. Allein die Schaffung einer neuen Institution schafft noch keine zusätzlichen Behandlungskapazitäten. Aber vielleicht ist die Diskussion um den zeitgerechten Zugang zur adäquaten Versorgung der Ausgangspunkt einer Debatte um die Schaffung bedarfsgerechter Kapazitäten und die patientenorientierte Steuerung von Behandlungsprozessen. Positiv ist in jedem Fall zu bewerten, dass in der psychotherapeutischen Versorgung künftig ein zeitnaher Zugang zur Kurzzeittherapie eröffnet und die Möglichkeiten für Gruppentherapie erweitert werden sollen. Damit wird adäquat auf die Enttabuisierung psychischer Erkrankungen und entsprechender Veränderungen im Nachfrageverhalten reagiert.

Aus Sicht der Versicherten besonders erfreulich ist die Schließung von Versorgungslücken beim Übergang von der stationären in die ambulante Versorgung. Dies gilt für das Entlassungsmanagement der Krankenhäuser ebenso wie für das Leistungsspektrum und die Koordinierungsaufgaben der Krankenkassen. Hier hat sich die Bremer Initiative Ambulante Versorgungslücken e.V. offenbar wirksam bemerkbar gemacht und den politischen Boden für eine Ausweitung von häuslicher Krankenpflege und Haushaltshilfe, für die Erweiterung von Verordnungsmöglichkeiten der Krankenhäuser bei der Entlassung und für eine aktivere Rolle der Krankenkassen im Versorgungsmanagement. Bleibt nur zu hoffen, dass nicht lobbyistisch geprägte Interessenpolitik und falsch verstandener Datenschutz die praktische Umsetzung dieser Vorgaben be- oder verhindern werden.

Für Menschen mit geistiger Behinderung und schweren Mehrfachbehinderungen soll die Versorgung verbessert und in Behandlungszentren analog den sozialpädiatrischen Zentren konzentriert werden. Dieser Schritt erscheint angesichts der Mängel und Lücken bei der medizinischen Versorgung Behinderter und Pflegebedürftiger in allen Bereichen dringend geboten. Er sollte auch genen den Widerstand der Vertragsärzteschaft umgesetzt werden. Den Versicherten kommt schließlich auch zugute, dass die strukturierten Behandlungsprogramme für chronisch Kranke (DMP), insbesondere bei Rückenleiden und Depressionen, ausgeweitet, die Freiräume der Krankenkassen im Wettbewerb mit Selektivverträgen ausgedehnt und die sektorübergreifende Qualitätssicherung ausgebaut werden.
Der Ausbau elektronischer Kommunikations- und Informationstechnologien sowie die Förderung und Vergütung der Telemedizin weisen neue Wege in der Versorgung - nicht nur in schwer zu versorgenden Regionen. Allerdings liegt hier der Teufel im Detail. Selbst klare gesetzliche Vorgaben mit Terminsetzung werden von den Kollektivvertragspartnern nicht umgesetzt, wenn sie ihren Partikularinteressen widersprechen. Hier wird der Gesetzgeber mit Ersatzvornahmen winken müssen, um der Einsichtsfähigkeit von Verbänden auf die Sprünge zu helfen. Ausgesprochen kontrovers diskutiert wird die Einrichtung eines Innovationsfonds in Höhe von 300 Mio. €, der je zur Hälfte von den Krankenkassen und dem Gesundheitsfonds gespeist wird. Positiv ist zu bewerten, dass eine systematische Förderung sektorübergreifender Versorgungsformen einschließlich der Evaluation nach vier Jahren und Versorgungsforschung erfolgen soll; Bedenken lassen sich allerdings aus dem bürokratischen Weg über den Gemeinsamen Bundesausschuss ableiten. Die Gefahr ist groß, dass dort diejenigen über Innovationen entscheiden sollen, die ein Interesse an der Festigung des Status Quo haben. Auch erscheint eine Ausschreibung unter dem Regime des Vergaberechts aufwändig und wenig innovationsfreundlich. Alternativ oder zumindest ergänzend hätte sich die Schaffung eines eigenen Budgets für Forschung und Entwicklung bei allen Akteuren des Gesundheitswesens angeboten, um einen Kulturwandel in den starren Strukturen herbeizuführen.

Man muss kein Anhänger des ordnungspolitischen Reinheitsgebots sein, um die mangelnde Kohärenz der Reformvorstellungen für die künftige Ausrichtung der Versorgungsstrukturen undprozesse zu beklagen. Einerseits sind die Koalitionäre bestrebt, Freiräume für die Akteure zu erweitern. Auch ist der Wille unverkennbar, wettbewerbsfreundliche Rahmenbedingungen zu schaffen. Exemplarisch seien hier nur die Angleichung bisher unterschiedlicher Rahmenbedingungen für selektive Vertragsbeziehungen in den $\mathbb{\int} \int 63-65,73 a-73 c, 140$ aff. SGB $\mathrm{V}$ und die Beseitigung von Hemmnissen und Diskriminierungen durch Aufsichtsbehörden und Kollektivvertragspartner genannt. Selbst eine Verpflichtung, nach einer Frist von vier Jahren Qualität und Wirtschaftlichkeit zu evaluieren und bei erfolgreichen Projekten den Übergang in 
die Regelversorgung zu prüfen, ist durchaus mit einer effektiven Wettbewerbsordnung zu vereinbaren. Die Krux liegt eher darin, dass kollektiven Steuerungsorganen und-systemen erneut neue Aufgaben und Funktionen zugewiesen werden, die Wettbewerb eher behindern als fördern.

\section{Ein kleiner Paradigmenwechsel in der Krankenhausversorgung - Vorrang für Qualität und Erreichbarkeit}

Ähnliche Zielsetzungen wie in der ambulanten Versorgung verfolgen die Koalitionsparteien in der stationären Versorgung-Qualität, Erreichbarkeit, Sicherheit. Die neue Mehrheit will eine Qualitätsoffensive starten und Qualität als neuen Parameter in der Krankenhausversorgung einführen. Die Arbeit des Qualitätsinstituts soll verstetigt und institutionell abgesichert werden. Die Richtlinienkompetenz des Gemeinsamen Bundesausschusses und die Kontrollbefugnisse des Medizinischen Dienstes sollen ausgeweitet werden. Die jährlichen Qualitätsberichte der Krankenhäuser sollen einheitlicher, ausführlicher und verständlicher werden, Aspekte der Patientensicherheit berücksichtigen und die Ergebnisse von Patientenbefragungen integrieren. Finanzielle Anreize zur Qualitätssteigerung sollen vor allem durch Ausnahmen von Mehrleistungsabschlägen gegeben werden. Geradezu revolutionär ist die Verständigung auf (zeitlich befristete) Qualitätsverträge zwischen einzelnen Krankenhäusern und einzelnen Krankenkassen. Da ist es auch zu verschmerzen, das allgemeine Kriterien gemeinsam und einheitlich auf Landesebne festzulegen sind. Sollte dies der Durchbruch zu Vertragswettbewerb im stationären Sektor sein? Zumindest ist es ein zartes Pflänzchen, das nicht sofort zertreten werden darf.

Besonders am Herzen liegt der Politik die Sicherstellung einer flächendeckenden Versorgung. Dazu sollen die Krankenhausplanung von einer Standortplanung auf eine Erreichbarkeitsplanung umgestellt und Sicherstellungszuschläge effektiver eingesetzt werden. Der Gemeinsame Bundesausschuss soll hierzu bundesweit gültige Kriterien entwickeln. In diesem Zusammenhang soll überprüft werden, ob die Teilnahme an der Notfallversorgung adäquat finanziert wird.

Seit längerem bestehen Zweifel, ob das DRG-System bei bestimmten Leis- tungen - vor allem bei Bandscheiben-OPs, Knie- und Hüftprothetik, Prostata-Entfernungen, Links-Herz-Katheter-Eingriffen nicht Anreize für eine medizinisch nicht indizierte Mengenausweitung bietet. Dem soll durch eine Verbesserung der Kalkulationsprozesse und durch ein versichertenfreundliches Zweitmeinungsverfahren begegnet werden. Speziell die Notfallversorgung außerhalb der Praxisöffnungszeiten in der ambulanten Versorgung sowie Hochkostenfälle in Universitätskliniken und Häusern der Maximalversorgung müssen sachgerechter vergütet werden, gegebenenfalls durch eine besondere Vergütungsform. Schließlich sollen das DRGSystem die Personalkosten, insbesondere der Pflege, besser abbilden und bei den Budgetverhandlungen auch deren tatsächliche Umsetzung kontrolliert werden.

Ein weiterer Schwerpunkt liegt bei der Gewährleistung der Patientensicherheit im Krankenhaus. Hierzu sind konkrete Verbesserungen bei der Einführung von Medizinprodukten mit hoher Risikoklasse, bei der Vermeidung von Krankenhausinfektionen und in der Transplantationsmedizin vorgesehen.

So überzeugend und umfassend die Reformvorstellungen in diesem ,zubetonierten" Versorgungsbereich auch sind, so ärgerlich ist der Wegfall eines Gemeinschaftsprogramms von Staat und Selbstverwaltung zur Um- und Abstrukturierung nicht bedarfsnotwendiger Kapazitäten. Dieses Programm ist dem Rotstift der Parteivorsitzenden und dem Irrtum zum Opfer gefallen, die Modernisierung der Infrastruktur in diesem Land ließe sich ohne Steuererhöhungen oder ohne Neuverschuldung finanzieren. Trotzdem sollte man die Hoffnung nicht aufgeben, dass die vereinbarte Bund-Länder-Arbeitsgruppe, die 2014 Eckpunkte für eine umfassende Krankenhausreform erarbeiten soll, diesen unter Fachplitikern bereits konsentierten Vorschlag wieder mit Leben erfüllen wird.

\section{Kontinuität in der \\ Arzneimittelversorgung - Nutzenbewertung und Preisverhandlungen als lernendes System}

Keine grundlegenden Umwälzungen wird es vorerst in der Arzneimittelversorgung geben. Die Nutzenbewertung für neue Produkte mit anschließenden Preisverhandlungen wird ebenso beibehalten wie das Preismoratorium und der (künftig auf
$7 \%$ reduzierte) Herstellerabschlag. Dafür wird auf den Aufruf des Bestandsmarkts verzichtet und dem GKV-Spitzenverband ein Placebo bei den Preisverhandlungen verordnet. Hier soll künftig ein in einem rechtssicheren Verfahren bestimmter Kassenvertreter beiwohnen. Dass die Rechtssicherheit für einen nahtlosen Übergang zum Jahreswechsel 2013/2014 gewährleistet wurde, hing vom sofortigen Beginn eines entsprechenden Gesetzgebungsverfahrens nach der Kanzlerwahl ab. Die Schließung der Lücken bei einer Indikationsausweitung lässt ein Hintertürchen für Produkte offen, die vor dem 1. Januar 2011 in den Markt eingeführt worden sind. Auch ist unklar, wie mit Produkten $\mathrm{zu}$ verfahren ist, für die die Nutzenbewertung vorliegt, die Preisverhandlungen einschließlich Schiedsverfahren zum Zeitpunkt des Inkrafttretens des Gesetzes noch nicht abgeschlossen sind. Der Koalitionsvertrag macht deutlich, dass er die AMNOG-Regelungen aus der letzten Legislaturperiode als lernendes System ansieht und bei Fehlentwicklungen und bei Gefährdung des Standorts Deutschland für Forschung und Produktion von Arzneimitteln korrigieren wird.

\section{Dritter Anlauf für ein Präventionsgesetz - Finanzierung unklar}

Bereits 2014 soll ein neuer Anlauf zu einem Präventionsgesetz erfolgen. Das Gesetz soll insbesondere lebensweltbezogene Settingansätze - so in Kitas, Schulen, Betrieben und Pflegeheimen - verfolgen und die betriebliche Gesundheitsförderung stärken. Konkrete Vorgaben zur finanziellen Absicherung dieser Aufgaben, die ursprünglich in den Vertragsentwurf aufgenommen waren, fielen in den Schlussrunden ebenfalls dem Rotstift zum Opfer. Trotzdem sollte man sich weiter dafür einsetzen, die inhaltlichen und finanziellen Freiräume für verhältnis- und verhaltensbezogene Prävention und für ein umfassend $\mathrm{zu}$ verstehendes betriebliches Gesundheitsmanagement zu erhöhen. Dann können alle Leistungsträger mehr Aktivitäten in allen Lebenswelten entfalten und die Reichweite ihrer Maßnbahmen beträchtlich erhöhen. Zentrale Ziele, die leider nicht im Koalitionsvertrag genannt sind, müssen dabei der Abbau gesundheitlicher und sozialer Benachteiligungen und damit die Verringerung gesundheitlicher Ungleichheiten sein. 


\section{Halbheiten bei der Umgestaltung des Finanzierungssystems - Festschreibung des \\ Arbeitgeberbeitrags, prozentualer Zusatzbeitrag für Versicherte und eine begrenzte Reform des Risikostrukturausgleichs}

Eine gemischte Bilanz ist bei der Bewertung des kurzen Abschnitts Finanzierung und Risikostrukturausgleich (RSA) zu ziehen. Unzweifelhaft sind die Umwandlung eines nominalen in einen prozentualen Zusatzbeitrag und die „gleichzeitige" Beseitigung von Methodenfehlern im morbiditätsorientierten Risikostrukturausgleich zwischen den Krankenkassen insgesamt positiv zu bewerten. Insbesondere um die Gleichzeitigkeit der Berücksichtigung der Kosten Verstorbener und die Korrektur von Verzerrungen beim Krankengeld wurde hart gerungen. Negativ fällt aber ins Gewicht, was nicht vereinbart wurde. Das gilt beispielsweise für die Wiedereinführung eines Risikopools für aufwändige Leistungsfälle, die vor allem kleinere und mittlere Krankenkassen vor existenzielle Probleme stellen, und die weiter gehende Überarbeitung des RSA im Hinblick auf Transparenz, einfache Handhabbarkeit und Verteilungsgerechtigkeit. Was nicht ist, kann ja bei der konkreten Umsetzung im Verlaufe des Jahres 2014 noch kommen.

Vielfältigen Protest hat die (erneuerte) Festschreibung des Arbeitgeberbeitrags zur Krankenversicherung entfacht. Es ist vollkommen unverständlich, warum sich Sozialdemokraten auf eine Verschlechterung gegenüber der automatischen Anpassungsklausel beim paritätischen Beitragssatz aus der letzten Großen Koalition eingelassen haben. Die Verhandlungsführer müssen die Unzulänglichkeit einer solchen Regelung geahnt haben, sonst hätten sie nicht in einer - zuerst unveröffentlichten - Nebenabrede festgehalten, dass sich die Finanzierungsanteile zwischen Versicherten und Arbeitgebern nicht $u$ ferlos auseinander entwickeln dürfen und deshalb die Fixierung auf diese Legislaturperiode begrenzt wird. Ein juristisch und politisch überaus zweifelhafter Vorgang, zumal er im Verabredungszeitraum keine Wirkung entfaltet.

Noch enttäuschender ist das völlige Fehlen von Abreden zur Zukunft der privaten Krankenversicherung und zur Konvergenz der Versicherungssysteme mit dem Ziel eines einheitlichen Kran- kenversicherungsmarkts, nachdem nicht nur die SPD das Ziel eines einheitlichen Krankenversicherungsmarkts postuliert hatte. Hier ist Lobbyeinfluss der auf die Kapitalrückstellungen als eigenes Vermögen schielenden Versicherungsunternehmen ebenso zu vermuten wie die (längst widerlegte) ordnungspolitisch angehauchte These vom Systemwettbewerb zwischen gesetzlicher und privater Versicherung. Nur Kinder können aber glauben, dass Probleme verschwinden, wenn man die Augen schließt. Der Druck der Verhältnisse wird voraussichtlich noch in dieser Legislaturperiode Änderungen an der Finanzverfassung und der Organisationstruktur des dualen Versicherungssystems erzwingen. Speziell der faktische Zwang für Beamte, sich privat zu versichern, ist ebenso fragwürdig wie der Ausschluss und die Begrenzungen bei der Übertragung von Alterungsrückstellungen, die ja wohl im Eigentum der Versicherten und nicht der Unternehmen stehen. Karlsruhe sollte bald Gelegenheit erhalten, hier für Klarstellung zu sorgen. MusterklägerInnen vor!

\section{Unklarheiten bei der Pflege - Wann soll was passieren?}

Ähnlich zwiespältig fällt die Bewertung der Reformvorstellungen im Bereich der Pflegeversicherung aus. Auch hier fehlt es an einem Zusammenwachsen von gesetzlicher und privater Versicherung, die sich nicht - wie ursprünglich vom Gesetzgeber erwartet-im Gleichklang entwickelt haben, sondern von einer ungleichen Verteilung der Pflegerisiken geprägt sind. Merkwürdig unpräzise sind zudem die (inhaltlichen und zeitlichen) Vorgaben für die Umsetzung des neuen Pflegebedürftigkeitsbegriffs. Diese soll „so schnell wie möglich“ erfolgen und speziell demenzielle Erkrankungen berücksichtigen, zuvor aber erprobt und wissenschaftlich ausgewertet werden. Ambitioniert klingen die vielfältigen Instrumente zur Verbesserung der (Arbeits-)Bedingungen von Pflegekräften, zur Förderung von Ehrenamt und Selbsthilfe sowie zur Ausweitung und besseren Abstimmung der Leistungen und vieles mehr.

Ob der anvisierte Finanzrahmen, der über eine zweistufige Anhebung des (paritätischen) Beitragssatzes um insgesamt 0,5 Beitragssatzpunkte finanziert werden soll, ausreicht, die Erwartungshaltungen der Pflegebedürftigen und ihrer Angehörigen, der Pflegeheime und Pflegedienste, aber vor allem der ehrenamtlichen und der professionellen Pflegenden gerecht zu werden, darf -bei allen vereinbarten Verbesserungen - schon heute bezweifelt werden. Am Grunddilemma der Pflegeversicherung als einer begrenzten Teilkaskoversicherung ändern auch diese Reformvorstellungen nichts. Um den Anforderungen des sozio-demografischen Wandels gerecht zu werden, muss der Umbau des Sozialsystems schneller und radikaler erfolgen.

\section{Fazit:}

Der Koalitionsvertrag zwischen Union und SPD beinhaltet keinen Politikwechsel in der Gesundheits- und Pflegepolitik, aber Fehlentwicklungen werden (zögerlich) korrigiert, Hindernisse für eine Weiterentwicklung des Bewährten beseitigt. Mutige Entwürfe, beispielsweise für eine sektorübergreifende Planung und Honorierung gesundheitlicher Leistungen, eine Verzahnung von Akutversorgung, Rehabilitation und Pflege oder einen integrierten Krankenversicherungsmarkt enthalten die Vereinbarungen nicht. Vielmehr bieten sie etwas mehr Chancen als Risiken für eine evolutionäre Weiterentwicklung tradierter Systeme. Ob wirklich die Patientinnen und Patienten künftig im Mittelpunkt von Politik und Steuerung des Gesundheitswesens stehen werden, muss weiterhin kritisch hinterfragt werden. Allerdings ist ein Koalitionsvertrag auch keine Bibel (so der frühere Bundeskanzler Gerhard

\section{Kein Politikwechsel zu} erwarten, aber Chancen zur Weiterentwicklung der Versorgung zu erkennen.

Schröder), sondern lediglich ein Fahrplan für eine Legislaturperiode. Auf neue Herausforderungen wird es Antworten geben müssen, die jetzt noch nicht absehbar sind. Auch wird die operative Umsetzung manchen Traum verblassen lassen. Trotzdem zeigen die Inhalte des Vertrages, dass auf die neue Mannschaft an der Spitze des Bundesgesundheitsministeriums und auf die Verantwortlichen in Bundestag und Bundesrat ein gefülltes Auftragsbuch wartet. Carpe diem! 